(2) Open Access Full Text Article

\title{
Assessing the prevalence of autoimmune, endocrine, gynecologic, and psychiatric comorbidities in an ethnically diverse cohort of female fibromyalgia patients: does the time from hysterectomy provide a clue?
}

This article was published in the following Dove Press journal: Journal of Pain Research

20 August 2015

Number of times this article has been viewed

\author{
Larry Brooks' \\ Joseph Hadi ${ }^{2}$ \\ Kyle T Amber ${ }^{1}$ \\ Michelle Weiner ${ }^{3}$ \\ Christopher L La Riche ${ }^{4}$ \\ Tamar Ference ${ }^{\prime}$ \\ 'Department of Rehabilitation \\ Medicine, University of Miami \\ Miller School of Medicine, Miami, \\ ${ }^{2}$ Anesco Interventional Pain Institute, \\ Margate, ${ }^{3}$ Miami Pain and Diagnostics, \\ Miami, ${ }^{4}$ Department of Psychiatry \\ and Behavioral Health, Florida \\ International University Wertheim \\ College of Medicine, University Park, \\ FL, USA
}

Background: This retrospective chart review investigated differences in the prevalence of medical comorbidity between women with fibromyalgia $(F M)(n=219)$ and a control group women with chronic pain $(\mathrm{CP})$ without FM $(\mathrm{n}=116)$. The specific aims were to compare the prevalence of autoimmune, psychiatric, endocrine, gynecologic pathology, the relationship between timing of gynecologic surgery, and pain onset. We additionally sought to compare the number of comorbidities in an ethnically diverse cohort.

Methods: This was a retrospective chart review of patients seen in FM or CP clinics at an academic medical center in 2009-2010.

Results: Logistic regression modeling found that gynecologic, endocrine, and autoimmune diagnoses were independently associated with a diagnosis of FM. Detailed analyses showed that thyroid disease $(P<0.01)$ and gynecologic surgery $(P<0.05)$ were significantly more common in FM. Women with FM were more likely to have multiple autoimmune, endocrine, gynecologic, or psychiatric pathologies. A relationship was observed between the timing of gynecologic surgery and pain onset in FM, with more surgeries observed in the years just prior to pain onset or in the year after pain onset. A similar pattern was not found in the control group.

Conclusion: This study demonstrates that autoimmune, endocrine, and gynecologic pathologies occur more commonly in women with FM than in those with CP, which is consistent with findings in less ethnically diverse samples. Moreover, a relationship was found between timing of pain onset and gynecologic surgery. A larger prospective study of the relationship between gynecologic surgery and pain onset in FM is warranted.

Keywords: fibromyalgia, sex hormones, thyroid, autoimmune, chronic pain

\section{Introduction}

Fibromyalgia (FM) has a total prevalence of $2 \%$ in the general population and is disproportionately more common in women after the age of 50 years, with a female to male ratio of $7: 1 .{ }^{1}$ In women, the prevalence of FM is only $0.9 \%$ between the ages of 18 and 30 years, but increases sharply at the age of 40-60 years, ${ }^{1}$ a period known for hormonal changes in women. A higher prevalence of pain in women compared with men is commonly reported across a number of pain conditions, including irritable bowel syndrome, ${ }^{2,3}$ temporomandibular disorder, ${ }^{4,5}$ chronic musculoskeletal pain, ${ }^{6}$ and migraines. ${ }^{7}$ FM has been demonstrated to have a higher degree of comorbidity with multiple conditions, including endocrine and genitourinary disorders, than in
Correspondence: Larry Brooks 3810 Hollywood Boulevard, Suite 2 Hollywood, FL 3302I, USA

Tel + I 9549623888

Fax + I 9549623936

Email lbrooksI@med.miami.edu 
osteoarthritis and other rheumatologic conditions. ${ }^{8,9} \mathrm{FM}$ frequently accompanies autoimmune disorders ${ }^{10,11}$ which also typically occur more commonly in women.

Several studies have assessed the presence of autoimmune disease in patients with FM and vice versa. There is an increase in autoimmune thyroid disease and autoantibody profile in FM patients, ${ }^{12}$ which has been proposed as a possible predisposing factor in the development of FM. ${ }^{13}$ The clinical severity of FM is more severe in patients with positive thyroid antibodies, and post-menopausal FM patients have a higher prevalence of thyroid antibodies than their premenopausal counterparts. ${ }^{11}$ Elevated thyroid antibodies are similarly seen in rheumatoid arthritis patients, which are also significantly greater than in healthy controls,${ }^{14}$ though some studies indicate a greater frequency of antibodies in rheumatoid arthritis patients than FM patients. ${ }^{15}$ There is additionally an increased prevalence of FM in systemic lupus erythematosus (SLE) patients and Sjogren patients, with one study demonstrating FM in 32\% of SLE patients and $18 \%$ of Sjogren patients. ${ }^{16} \mathrm{FM}$ is associated with worse health status and an increase in psychiatric symptoms in SLE patients. ${ }^{17}$ Interestingly, when analyzing the converse, there appears to be no increase in the risk of developing SLE in patients previously diagnosed with FM. ${ }^{13}$ Yet, there is a higher prevalence of Raynaud's phenomenon, sicca symptoms, and autoantibodies in FM patients. ${ }^{18}$

The sex differences in the prevalence of chronic pain (CP) conditions have generated multiple review articles about the role of sex hormones in pain onset, maintenance, or inhibitory control. ${ }^{19-24}$ Though substantial evidence links gonadal hormones and pain, the relationship between gonadal hormones and pain is not fully understood. One study showed that estrogen may have pronociceptive or antinociceptive effects depending on the receptor. ${ }^{25}$ Sex steroids alter levels of neuromodulators involved in spinal nociceptive processing, including substance $\mathrm{P}$, amino acids such as gamma aminobutyric acid, and glutamate and have been shown to increase the number of excitatory synapses in neurons in the cortex, cerebellum, and hippocampus. ${ }^{24}$ Several studies have examined the relationship of gynecologic pathology to FM. Some (but not all) studies have shown fluctuations in pain levels depending on the menstrual cycle phase. ${ }^{20,26,27}$ Hysterectomies and early menopause additionally occur more commonly in FM patients than in rheumatoid arthritis controls. ${ }^{28,29}$ Interestingly, Warren et al have noted an increased frequency of hysterectomies in patients developing bladder pain syndrome, ${ }^{30}$ an entity associated with the incidence of FM. ${ }^{31}$ Further, women with FM who had a hysterectomy with or without oophorectomy were recently shown to have worse symptom severity than those without hysterectomy. ${ }^{32}$ In an additional study of female FM patients, not only was there a greater frequency of women who had received a hysterectomy versus those who had not, but $90 \%$ of the FM patients with hysterectomies reported receiving the surgery before the onset of FM. ${ }^{33}$ Having FM with a previous history of a hysterectomy was associated with a lower general health status. ${ }^{32}$

In addition to sex hormones, research has implicated other endocrine pathologies, such as those involving the the hypothalamic-pituitary-adrenal (HPA) axis $^{34-37}$ and thyroid ${ }^{8,9,11,38-41}$ in FM. Research on the HPA axis in FM has shown variations in cortisol levels, ${ }^{42,43}$ increased sensitivity to glucocorticoid feedback, ${ }^{44}$ and increased cortisol release in response to a stressor. ${ }^{45}$ However, other studies have failed to find evidence of cortisol dysregulation in FM. ${ }^{36}$ Following a 3-week, multidisciplinary, FM treatment program, improvements were noted in the function of HPA axis, specifically cortisol and corticoid receptor levels. ${ }^{46}$ Altogether, the literature suggests that cortisol release may be abnormal in FM, but the nature of the pathology remains poorly understood at present.

In light of these previous studies, we sought to confirm these findings in our cohort of female patients, analyzing whether patients with FM had more autoimmune, endocrine, or gynecologic pathology than women of similar age with $\mathrm{CP}$ of a similar duration. As recent studies of medical comorbidities and FM included ethnically homogenous populations, with $97.4 \%$ of patients being non-Hispanic White, we sought to confirm these findings in a more ethnically diverse population. ${ }^{8} 9$ Specifically, this FM cohort is 53.8\% Hispanic. We additionally sought to provide more detailed information on specific diagnoses and procedures, such as hysterectomies and oophorectomies, than previous studies on medical comorbidities in FM. ${ }^{8,9}$ We examined the relationship between onset of pain and the onset of other disorders. This was accomplished by looking at the timing of pain onset and date of hysterectomy or oophorectomy. In addition to endocrine and gynecologic variables, we also measured the prevalence of other disorders frequently comorbid with FM, such as psychiatric illness, chronic fatigue syndrome, and irritable bowel syndrome.

\section{Materials and methods \\ Screening}

We screened all patients seen in 2009-2010 in a specialty FM clinic and in a CP management clinic at an academic medical 
center for inclusion in the study. All patients who saw either of the two physicians in the FM clinic or general CP clinic were screened electronically. Patients were excluded if they were men or had a cancer-related pain condition as their primary diagnosis. A second group of patients was excluded if they had pain for less than 6 months, a myofascial pain component without an FM diagnosis, or a medical record with insufficient documentation regarding pain diagnosis. Myofascial pain was used as an exclusion criterion due to concern over overlap with FM. The most common diagnoses among patients with CP included osteoarthritis and lower back pain. These patients did not meet the criteria for FM. A diagnosis of FM was made based on the previously published American College of Rheumatology 1990 criteria which includes the use of tender points. ${ }^{47}$ Approval to conduct this study was granted by the institutional review board of the University of Miami. A waiver of informed consent was granted for this retrospective chart review.

\section{Chart review}

Charts were reviewed by the principal author (LB) and three medical residents. All charts reviewed by the residents were reviewed at least twice, and inconsistencies between reviewers were identified and resolved by re-reviewing the charts. Ten percent of the charts reviewed by LB and by a resident were assessed for interrater reliability, which exceeded $90 \%$.

For each participant, data were gathered on diagnosis, year of pain onset, medical comorbidities, psychiatric comorbidities, surgical history, and date of hysterectomy, oophorectomy, or cystectomy. In addition to obtaining information from the physician's notes, information on specific aspects of the medical history (previous surgeries, dates of surgeries, date of pain onset, and psychiatric diagnoses) from self-report medical history questionnaires was used when available. The diagnosis of FM was based on the physician note, while self-report medical history questionnaires and registration papers used for patients enrolling in the clinic, provided additional past medical history or clarifications of the physician note. The most difficult information to collect was the date of pain onset and dates of surgical procedures due to a lack of documentation. There was a special issue with the psychiatric data, as there were concerns that it was assessed with greater frequency as a matter of clinical practice in the FM clinic. Due to concerns over potential bias regarding the psychiatric data, all analyses in this study were run with and without the psychiatric data included. Though data were collected on most medical comorbidities, the focus of data collection was on endocrine, autoimmune, gynecologic, and psychiatric diagnoses. Grouping of diagnoses into variables is delineated in Table 1. Gynecologic surgeries potentially involving the ovaries were analyzed individually and collectively to account for heterogeneity of documentation (eg, hysterectomy, hysterectomy + oophorectomy, and hysterectomy + oophorectomy + cystectomy). This analysis additionally allowed for a progressively more inclusive method for identifying gynecologic procedures, with hysterectomy + oophorectomy + cystectomy being the most inclusive cohort.

Data were also collected on hypertension, cholecystectomy, and conditions frequently comorbid with FM, such

Table I Diseases included in each pathology grouping

\begin{tabular}{lll}
\hline Variable & Chart diagnoses included & \\
\hline Autoimmune & Systemic lupus erythematosus, Rheumatoid arthritis, & Scleroderma, Idiopathic thrombocytopenic purpura, \\
& $\begin{array}{l}\text { Sjogren's syndrome, Multiple sclerosis, CREST, } \\
\text { Polymyalgia rheumatica } \\
\text { Hashimoto's disease, Hypothyroidism, Grave's disease, }\end{array}$ & anemia \\
Total thyroid & Hyperthyroidism & Goiter, Thyroid nodule, Thyroidectomy \\
Total psychiatric & Depression, Anxiety & Schizoaffective disorder, Schizophrenia \\
Total endocrine & Pituitary microadenoma, Hypopituitarism, Adrenalectomy, & Parathyroid adenoma, Pineal tumor, Neuroendocrine \\
& Adrenal insufficiency, Parathyroidectomy & tumor \\
Total gynecologic & Hysterectomy, Oophorectomy, Endometriosis, & Ovarian cystectomy, Myomectomy, Uterotomy, \\
& Endometrial hyperplasia, Polycystic ovarian syndrome, & Endometrial ablation, Cervical polyp removal, \\
& Uterine fibroid, Fibroid removal, Ovarian cyst rupture & Uterine cancer, Uterine rupture \\
Gynecologic surgery & Hysterectomy, Oophorectomy, Fibroid removal, & Uterotomy, Endometrial ablation, Cervical polyp removal \\
\end{tabular}

Abbreviations: CREST, calcinosis, Raynaud's phenomenon, esophageal dysmotility, sclerodactyly, and telangiectasia. 
as chronic fatigue syndrome and irritable bowel syndrome. Hypertension and cholecystectomy were selected because they are commonly occurring conditions and were mostly unrelated to the hypothesized relationships in the study, and thus serve as a form of control variable to assess whether or not patients with FM had more of all diagnoses or if the increased diagnostic burden was specific to the hypothesized conditions.

\section{Statistical analyses}

All statistical analyses in the study were made using Statistical Package for the Social Sciences for Windows version 19.0 software (IBM Corporation, Armonk, NY, USA). Two logistic regression analyses were run. The first logistic regression model included three binary, categorical, predictor variables: history of autoimmune disorder, history of endocrine disorder, and history of gynecologic disorder of surgery. The second model included these three variables plus a history of psychiatric disorder. All variables were included in a single block with forced entry into the model.

In essence, the logistic regression analyses served as omnibus tests to determine if autoimmune, endocrine, gynecologic, and psychiatric disorders were independently associated with the diagnosis of FM. Chi-square analyses were used to look with more detail at the difference in prevalence of disease pathology across groups. The significance of these tests was defined as $P \leq 0.05$ for a priori tests and as $P \leq 0.01$ for post hoc tests to correct for multiple comparisons. The relationship between the timing of pain onset and the timing of hysterectomy/oophorectomy was analyzed for the subset of patients for whom such data were available $(n=93)$. Additionally, Mann-Whitney $U$ tests were used to assess if there were differences in the amount of comorbidities between FM and CP participants.

\section{Results}

A total of 335 female patients were included in the study, with 219 in the FM group and 116 in the CP group. After excluding the nine patients who were not categorized as Caucasian, African-American, or Hispanic, analyses revealed significant differences in the ethnic composition of the FM and CP groups $\left(\chi^{2}=17.6 ; P<0.001\right)$. The percentage of Caucasian patients as a total of the FM and CP groups was consistent (34.2\% versus $34.9 \%$, respectively). However, a larger percentage of Hispanics were in the FM group than in the CP group (53.8\% versus $36.8 \%$, respectively). In contrast, more African-Americans were members of the CP group than of the FM group ( $28.9 \%$ versus $11.3 \%$, respectively). The mean (standard deviation) age for the FM group was 52.66 (12.3) and that of the CP group was 52.5 (13.3). No differences between groups were found by age $(P=0.88)$ or years since pain onset $(P=0.55)$.

\section{Logistic regression modeling}

In the first logistic regression model, all three variables (autoimmune, total endocrine, total gynecologic) were significantly associated with the FM diagnosis (Table 2). When psychiatric history was included in the model, a history of autoimmune or endocrine disorders continued to be independently associated with a diagnosis of FM; however, a history of a gynecologic disorder or surgery did not remain significant.

\section{Medical comorbidities}

Autoimmune disorders, hypothyroidism, total thyroid, total endocrine, total gynecologic, depression, and total diseases included in each pathology grouping were significantly more frequent in the FM group. No differences in diabetes and hypertension were found between groups. Chronic fatigue syndrome and irritable bowel syndrome were more common in the FM than in the CP group. Exact proportions and gross $P$-values are listed in Table 3.

\section{Surgical history and relationship of hysterectomy/oophorectomy with pain onset}

In terms of surgical history, hysterectomy + oophorectomy, hysterectomy + oophorectomy + cystectomy, and total gynecologic surgeries occurred more frequently in the FM group. Across all participants with a history of hysterectomy or oophorectomy, the procedure preceded pain onset in $73.1 \%$ of cases. On average, surgery occurred 8.3 (12.8) years before pain onset. The range was from surgery occurring 43 years before pain onset to surgery occurring 19 years

Table 2 Logistic models predicting a diagnosis of fibromyalgia

\begin{tabular}{llll}
\hline & $\beta$ & OR (95\% CI) & P-value \\
\hline Model I & & & \\
Autoimmune & 0.83 & $2.29(\mathrm{I} .12-4.70)$ & 0.02 \\
Total endocrine & 0.82 & $2.27(\mathrm{I} .26-4.10)$ & 0.007 \\
Total gynecologic & 0.48 & $\mathrm{I} .62(\mathrm{I} .02-1.59)$ & 0.04 \\
Model 2 & & & \\
Autoimmune & $\mathrm{I} .10$ & $3.0 \mathrm{I}(\mathrm{I} .40-6.43)$ & 0.005 \\
Total endocrine & 0.79 & $2.2 \mathrm{I}(\mathrm{I} .19-4.1 \mathrm{I})$ & $0.0 \mathrm{I}$ \\
Total gynecologic & 0.28 & $\mathrm{I} .32(0.80-2.19)$ & 0.27 \\
Total psychiatric & $\mathrm{I} .49$ & $4.45(2.69-7.35)$ & 0.001 \\
\hline
\end{tabular}

Abbreviations: $\mathrm{Cl}$, confidence interval; OR, odds ratio. 
Table 3 Proportion of patients with comorbidities by group

\begin{tabular}{|c|c|c|}
\hline & $\begin{array}{l}\text { Fibromyalgia } \\
(n=219)\end{array}$ & $\begin{array}{l}\text { Chronic pain } \\
(n=I \mid 6)\end{array}$ \\
\hline \multicolumn{3}{|c|}{ Endocrine and autoimmune comorbidities } \\
\hline Autoimmune disorders & $19.2 \%$ & $9.5 \% *$ \\
\hline Endocrine + thyroid + diabetes & $44.7 \%$ & $34.6 \% *$ \\
\hline Diabetes & $24.2 \%$ & $19.8 \%$ \\
\hline Any thyroid & $27.4 \%$ & $14.7 \% * *$ \\
\hline Hypothyroid & $21.5 \%$ & $11.2 \% *$ \\
\hline Thyroidectomy & $4.6 \%$ & $1.7 \%$ \\
\hline Endocrine + thyroid & $30.1 \%$ & $15.5 \% * *$ \\
\hline Endocrine (no thyroid) & $4.1 \%$ & $1.7 \%$ \\
\hline \multicolumn{3}{|l|}{ Gynecologic comorbidities } \\
\hline $\begin{array}{l}\text { Any gynecologic surgery or } \\
\text { illness }\end{array}$ & $54.8 \%$ & $39.7 \% * *$ \\
\hline Any gynecologic surgery & $51.6 \%$ & $38.8 \% *$ \\
\hline Hysterectomy & $39.3 \%$ & $30.2 \%$ \\
\hline Hysterectomy/oophorectomy & $43.4 \%$ & $30.2 \% *$ \\
\hline $\begin{array}{l}\text { Hysterectomy/oophorectomy/ } \\
\text { cystectomy }\end{array}$ & $45.7 \%$ & $31.9 \% *$ \\
\hline \multicolumn{3}{|l|}{ Other comorbidities } \\
\hline Hypertension & $45.7 \%$ & $42.2 \%$ \\
\hline Cholecystectomy & $17.8 \%$ & $16.4 \%$ \\
\hline Irritable bowel syndrome & $9.6 \%$ & $0.9 \% * *$ \\
\hline Chronic fatigue syndrome & $5.5 \%$ & $0.0 \% * *$ \\
\hline \multicolumn{3}{|l|}{ Psychiatric comorbidities } \\
\hline $\begin{array}{l}\text { Depression + severe mental } \\
\text { illness }\end{array}$ & $71.7 \%$ & $37.1 \% * *$ \\
\hline Depression & $66.2 \%$ & $35.3 \% * *$ \\
\hline
\end{tabular}

Notes: $* P \leq 0.05 ; * * P \leq 0.01$.

after pain onset. When the distributions between FM and CP groups are displayed, there is a noticeable difference in the shape of the distributions (Figures 1 and 2). Of note, 25 and 12 patients were excluded from the FM group and control group, respectively, as the time of hysterectomy was not available in the medical records.
In the FM group, the peak of the distribution occurs with surgeries occurring in the 4 (mean 7.7, median 3.5) years just before pain onset or in the year after pain onset (Figure 1). That perioperative time period accounts for $47.1 \%$ of the hysterectomies and oophorectomies in FM patients. In contrast, the same period accounts for $24.0 \%$ of surgeries in CP patients (mean 9.1, median 12 years). In contrast with the FM patients, the distribution of the timing between pain onset and time of surgery in CP patients appears to be more random.

\section{Aggregates across domains}

Aggregates for each participant were calculated for endocrine, gynecologic, and autoimmune disorders. Data were coded in a binary fashion for each type of disorder (endocrine, gynecologic, and autoimmune) for this analysis. The maximum potential score was 3 . For instance, if a participant had an autoimmune diagnosis and an endocrine diagnosis, then the subject would receive a score of 2 . An individual with two endocrine diagnoses would only receive a score of 1 . An additional set of analyses was conducted including endocrine, gynecologic, and autoimmune plus psychiatric diagnosis. Mann-Whitney $U$ tests showed significant differences between the FM and CP groups, both when the analyses included only endocrine, gynecologic, and autoimmune diagnoses $(U=9,339 ; P<0.001)$ and also when psychiatric diagnoses were included $(U=7,556 ; P<0.001)$. Individuals in the FM group were 2.4 times more likely to have more than one endocrine, gynecologic, or autoimmune diagnosis (see Figure 3). When including psychiatric diagnoses, individuals in the FM group were 4.0 times more likely to have three or more diagnoses (Figure 4).

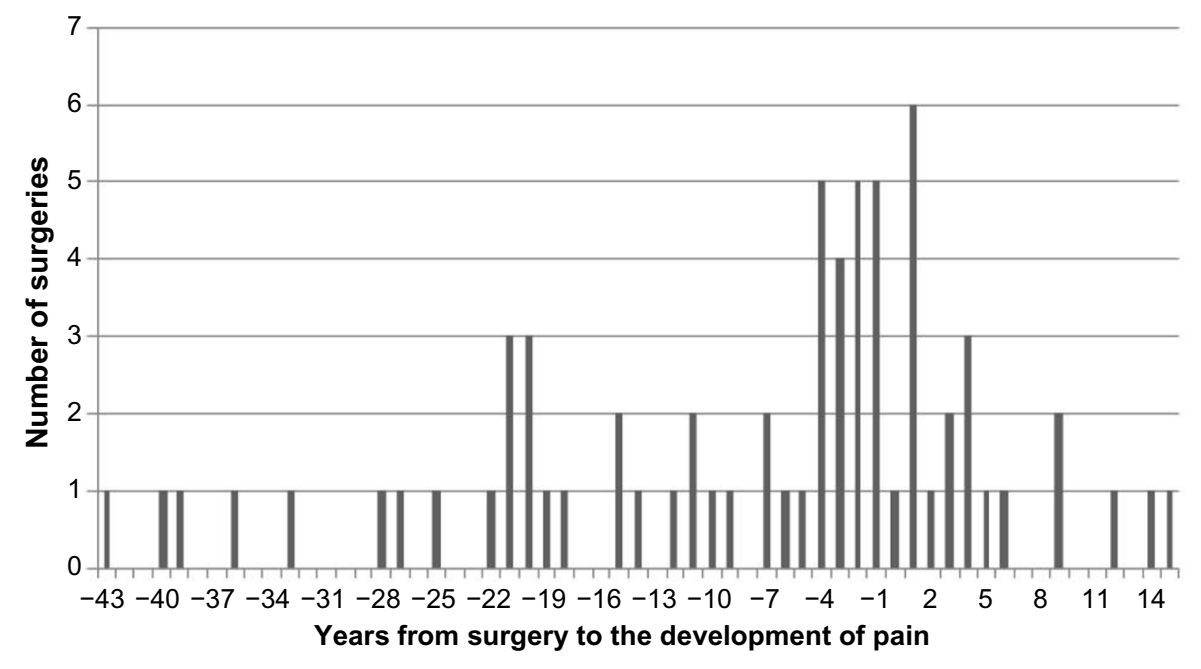

Figure I Hysterectomy/oophorectomy and pain onset in fibromyalgia $(n=68)$. 


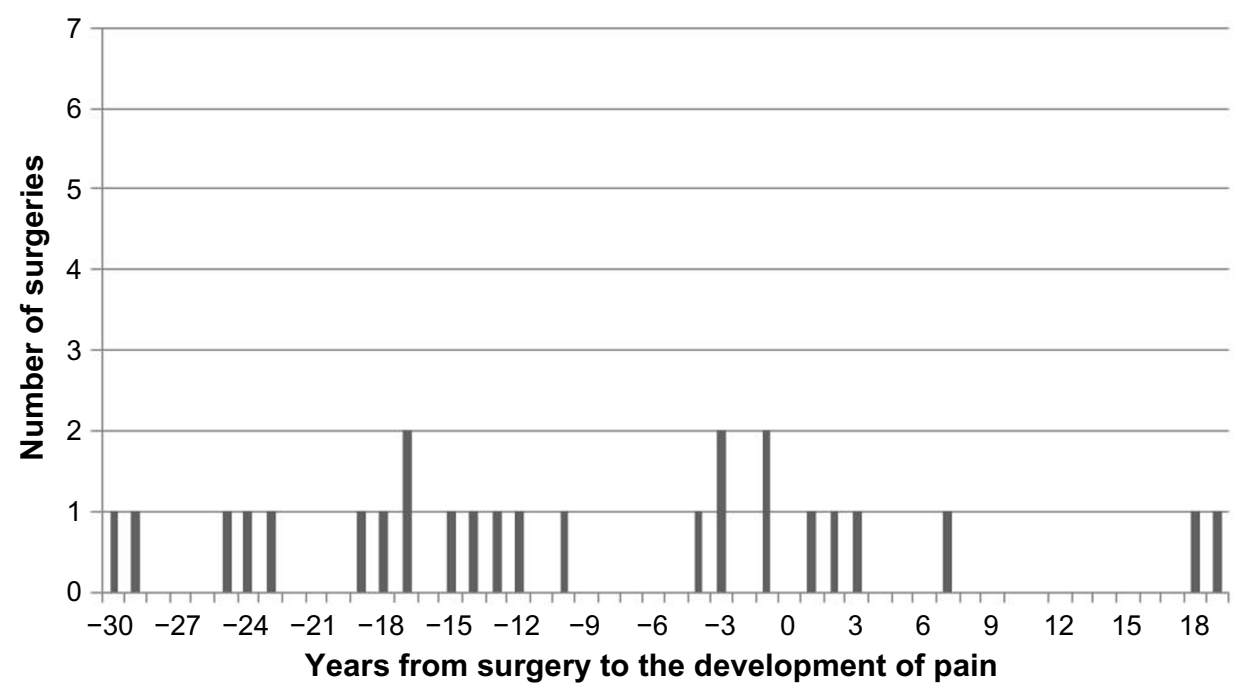

Figure 2 Hysterectomy/oophorectomy and pain onset in chronic pain $(n=25)$.

\section{Discussion}

This study confirmed previous findings that autoimmune, gynecologic, endocrine, and total medical comorbidities are elevated in women with FM, even in an ethnically diverse cohort. We additionally demonstrate that there may be a temporal relationship between pain onset and timing of hysterectomy/oophorectomy. Further, we found that carrying multiple diagnoses was more common in the FM group than in the control group. As our control group consisted of women of a similar age with CP for a similar amount of time, differences between the groups may be inferred to be disease-related and not age-related or pain-related. Our control cohort consisted of CP patients, a majority of whom had lower back pain and/ or osteoarthritis, as opposed to rheumatoid arthritis patients as in previous studies. Given the potentially important role of autoimmunity in the pathogenesis of $\mathrm{FM}^{48}$ a $\mathrm{CP}$ cohort likely serves as a more meaningful control than a rheumatoid arthritis control group.

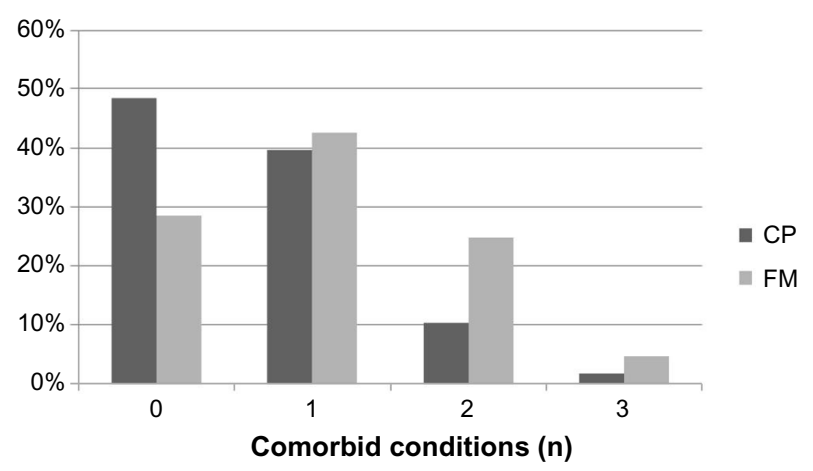

Figure 3 Number of comorbid endocrine, gynecologic, and autoimmune conditions.

Abbreviations: $\mathrm{CP}$, chronic pain; FM, fibromyalgia.
Our results regarding the increased prevalence of gynecologic, endocrine, and psychiatric disorders were remarkably similar to the findings of disease prevalence reported by Wolfe et al. ${ }^{9}$ This similarity in prevalence is more noteworthy given the demographic differences in ethnicity between the two study populations, thereby increasing the generalizability of these findings. Altogether, the data suggest that our study's findings of increased endocrine, autoimmune, gynecologic, and psychiatric comorbidities in FM are specific and not due to age, ethnicity, or an increase in all diagnoses or procedures. The finding that hysterectomies are more common in women with FM has been demonstrated previously. ${ }^{28,29}$ Likewise, hysterectomies were related to greater symptom severity. ${ }^{32}$

Hysterectomies are the second most common surgery among reproductive-aged women, ${ }^{49}$ with more than onethird of US women having had a hysterectomy by the age of 60 years. ${ }^{50}$ Therefore, if hysterectomies are a contributor

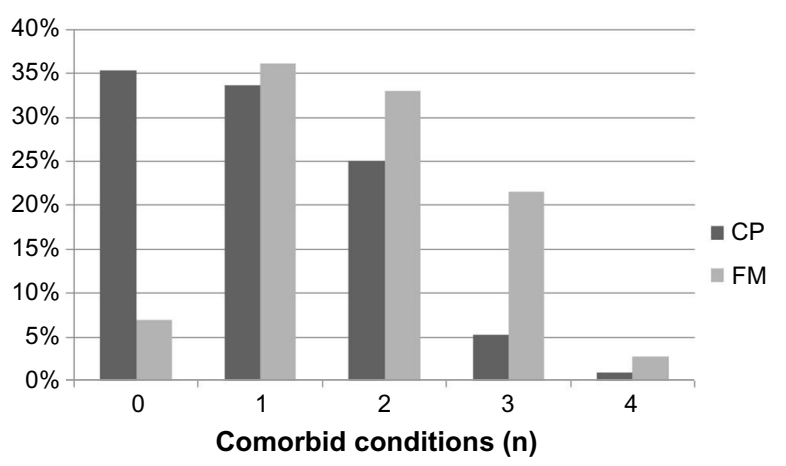

Figure 4 Number of comorbid endocrine, gynecologic, autoimmune, and psychiatric conditions.

Abbreviations: $\mathrm{CP}$, chronic pain; FM, fibromyalgia. 
to the development of FM, then why is the prevalence of FM so much lower than the prevalence of hysterectomies? Though genetic differences or pre-disease environmental factors likely play a role in who develops FM, one possible answer to the question of who develops FM is supported by the cumulative burden data. The data show that the presence of only one autoimmune, endocrine, or gynecologic condition is no more common in FM than in the CP control group; however, participants who had more than one condition were significantly more likely to have FM. This finding is even more pronounced when the psychiatric data are included as well.

An alternative explanation for our study findings is that a common cause is responsible for the increase in autoimmune, gynecologic, endocrine, and psychiatric comorbidities. For instance, dysfunction at the level of the hypothalamus or the pituitary could be responsible for most of those disorders. Previous review articles have documented that hypothalamic-pituitary dysfunction is implicated in gynecologic $c^{51,52}$ and psychiatric disorders, ${ }^{53,54}$ while there additionally appears to be HPA axis abnormalities in autoimmune disease. ${ }^{55,56}$ By definition, dysfunction at the level of the hypothalamus or the pituitary involves endocrine pathology. Central pathology of the hypothalamus or pituitary could explain some of the frequent symptoms in FM involving sleep pathology, ${ }^{57,58}$ fatigue, ${ }^{57}$ and cognitive complaints, ${ }^{58,59}$ as all have been related to hypothalamic or pituitary dysfunction or the downstream effects of such dysfunction.

The increased amount of gynecologic pathology in FM, as well as the relationship between the timing of gynecologic surgery and pain onset, indicates that a hypoestronergic state may contribute to increased pain perception. Despite striking sex differences in the prevalence of FM and other $\mathrm{CP}$ conditions, the effects of estrogen on pain perception are poorly understood. A relatively recent review of estrogen and pain highlighted the effects of estrogen on multiple sites and systems involved in nociceptive processing, including the hypothalamus, pituitary gland, descending noxious inhibitory controls, sympathetic nervous system, primary afferents, and the dorsal horn of the spinal cord ${ }^{60}$ Estrogen has also been shown to modulate the capsaicin (TRPV1) receptor ${ }^{61,62}$ and the dorsal root ganglia. ${ }^{63}$ Data on the peak prevalence of FM approximately overlap with menopause, with one study showing a significant increase in prevalence in women after the age of 50 years, ${ }^{1}$ and a second study showing a peak prevalence between 55 and 64 years. ${ }^{64}$ Thus, further study of the relationship between estrogen and FM is warranted.
The primary limitation of the study is the limited standardization of the manner in which data were collected in the two separate pain clinics due to the study's retrospective nature. In both clinics, the nursing staff take a medical history in a similar fashion; however, there is more of a focus on psychiatric comorbidity in the FM clinic. Consequently, the identified differences between the two groups may be an artifact of a lack of standardization in data collection methods, particularly with respect to psychiatric data. The most likely flaw would be an underrepresentation of psychiatric illness in one of the groups due to insufficient assessment. This is the reason that all data are presented in this paper with and without psychiatric data. It should be noted that the rates of depression found in the participants are generally consistent with a recent study of comorbidities in FM, ${ }^{9}$ which lends greater credence to the psychiatric data presented in the paper. Additionally, while both clinics diagnosed FM based on the American College of Rheumatology criteria from 1990 that uses tender points as a means of making a diagnosis, the tender point method is not an ideal means of diagnosing FM and has thus been replaced in more recent proposed guidelines. ${ }^{65} \mathrm{An}$ additional limitation is that it was often difficult to find the desired information. For instance, the data related to the timing of surgery in relation to pain onset could only be identified in approximately half of the patients. Further, information related to hysterectomy was limited, such that it was impossible to determine if ovaries were conserved or removed. This lack of specific documentation occurred in both groups and thus likely did not introduce bias. Similarly, the use of patient reported pain onset is not an entirely reliable outcome measure. In the future, a prospective study with standardized data collection methods including detail as to the timing and nature of surgical procedures will allow for more thorough study of the issues raised in this paper.

\section{Disclosure}

The authors report no conflicts of interest in this work.

\section{References}

1. Wolfe F, Ross K, Anderson J, Russell IJ, Hebert L. The prevalence and characteristics of fibromyalgia in the general population. Arthritis Rheum. 1995;38(1):19-28.

2. Drossman DA, Li Z, Andruzzi E, et al. US householder survey of functional gastrointestinal disorders. Prevalence, sociodemography, and health impact. Dig Dis Sci. 1993;38(9):1569-1580.

3. Sandler RS. Epidemiology of irritable bowel syndrome in the United States. Gastroenterology. 1990;99(2):409-415.

4. Riley JL 3rd, Gilbert GH. Orofacial pain symptoms: an interaction between age and sex. Pain. 2001;90(3):245-256.

5. Rauhala K, Oikarinen KS, Jarvelin MR, Raustia AM. Facial pain and temporomandibular disorders: an epidemiological study of the Northern Finland 1966 Birth Cohort. Cranio. 2000;18(1):40-46. 
6. Tsang A, Von Korff M, Lee S, et al. Common chronic pain conditions in developed and developing countries: gender and age differences and comorbidity with depression-anxiety disorders. J Pain. 2008; 9(10):883-891.

7. Lipton RB, Stewart WF, Diamond S, Diamond ML, Reed M. Prevalence and burden of migraine in the United States: data from the American Migraine Study II. Headache. 2001;41(7):646-657.

8. Wolfe F, Hawley DJ. Evidence of disordered symptom appraisal in fibromyalgia: increased rates of reported comorbidity and comorbidity severity. Clin Exp Rheumatol. 1999;17(3):297-303.

9. Wolfe F, Michaud K, Li T, Katz RS. Chronic conditions and health problems in rheumatic diseases: comparisons with rheumatoid arthritis, noninflammatory rheumatic disorders, systemic lupus erythematosus, and fibromyalgia. J Rheumatol. 2010;37(2):305-315.

10. Crosslin KL, Wiginton KL. Sex differences in disease severity among patients with systemic lupus erythematosus. Gend Med. 2011; 8(6):365-371.

11. Bazzichi L, Rossi A, Giuliano T, et al. Association between thyroid autoimmunity and fibromyalgic disease severity. Clin Rheumatol. 2007; 26(12):2115-2120.

12. Suk JH, Lee JH, Kim JM. Association between thyroid autoimmunity and fibromyalgia. Exp Clin Endocrinol Diabetes. 2012;120(7):401-404.

13. Kotter I, Neuscheler D, Gunaydin I, Wernet D, Klein R. Is there a predisposition for the development of autoimmune diseases in patients with fibromyalgia? Retrospective analysis with long term follow-up. Rheumatol Int. 2007;27(11):1031-1039.

14. Pamuk ON, Cakir N. The frequency of thyroid antibodies in fibromyalgia patients and their relationship with symptoms. Clin Rheumatol. 2007;26(1):55-59.

15. Shiroky JB, Cohen M, Ballachey ML, Neville C. Thyroid dysfunction in rheumatoid arthritis: a controlled prospective survey. Ann Rheum Dis. 1993;52(6):454-456.

16. Iannuccelli C, Spinelli FR, Guzzo MP, et al. Fatigue and widespread pain in systemic lupus erythematosus and Sjogren's syndrome: symptoms of the inflammatory disease or associated fibromyalgia? Clin Exp Rheumatol. 2012;30(6 Suppl 74):117-121.

17. Torrente-Segarra V, Carbonell-Abello J, Castro-Oreiro S, Manresa Dominguez JM. Association between fibromyalgia and psychiatric disorders in systemic lupus erythematosus. Clin Exp Rheumatol. 2010;28(6 Suppl 63):S22-S26.

18. Dinerman H, Goldenberg DL, Felson DT. A prospective evaluation of 118 patients with the fibromyalgia syndrome: prevalence of Raynaud's phenomenon, sicca symptoms, ANA, low complement, and Ig deposition at the dermal-epidermal junction. J Rheumatol. 1986;13(2):368-373.

19. Manson JE. Pain: sex differences and implications for treatment. Metabolism. 2010;(59 Suppl 1):S16-S20.

20. Martin VT. Ovarian hormones and pain response: a review of clinical and basic science studies. Gend Med. 2009;(6 Suppl 2):168-192.

21. Fillingim RB, King CD, Ribeiro-Dasilva MC, Rahim-Williams B, Riley JL 3rd. Sex, gender, and pain: a review of recent clinical and experimental findings. J Pain. 2009;10(5):447-485.

22. Craft RM, Mogil JS, Aloisi AM. Sex differences in pain and analgesia: the role of gonadal hormones. Eur J Pain. 2004;8(5):397-411.

23. Fillingim RB, Ness TJ. Sex-related hormonal influences on pain and analgesic responses. Neurosci Biobehav Rev. 2000;24(4):485-501.

24. Aloisi AM, Bonifazi M. Sex hormones, central nervous system and pain. Horm Behav. 2006;50(1):1-7.

25. Coulombe MA, Spooner MF, Gaumond I, Carrier JC, Marchand S. Estrogen receptors beta and alpha have specific pro- and anti-nociceptive actions. Neuroscience. 2011;184:172-182.

26. Riley JL 3rd, Robinson ME, Wise EA, Price DD. A meta-analytic review of pain perception across the menstrual cycle. Pain. 1999;81(3): 225-235.

27. Hellstrom B, Anderberg UM. Pain perception across the menstrual cycle phases in women with chronic pain. Percept Mot Skills. 2003; 96(1):201-211.
28. ter Borg EJ, Gerards-Rociu E, Haanen HC, Westers P. High frequency of hysterectomies and appendectomies in fibromyalgia compared with rheumatoid arthritis: a pilot study. Clin Rheumatol. 1999;18(1):1-3.

29. Pamuk ON, Donmez S, Cakir N. Increased frequencies of hysterectomy and early menopause in fibromyalgia patients: a comparative study. Clin Rheumatol. 2009;28(5):561-564.

30. Warren JW, Howard FM, Morozov VV. Is there a high incidence of hysterectomy and other nonbladder surgeries before and after onset of interstitial cystitis/bladder pain syndrome? Am J Obstet Gynecol. 2013;208(1):77. e71-e76.

31. Martinez-Martinez LA, Mora T, Vargas A, Fuentes-Iniestra M, Martinez-Lavin M. Sympathetic nervous system dysfunction in fibromyalgia, chronic fatigue syndrome, irritable bowel syndrome, and interstitial cystitis: a review of case-control studies. J Clin Rheumatol. 2014;20(3):146-150.

32. Vincent A, Whipple MO, Luedtke CA, et al. Pain and other symptom severity in women with fibromyalgia and a previous hysterectomy. J Pain Res. 2011;4:325-329.

33. Santoro MS, Cronan TA, Adams RN, Kothari DJ. Fibromyalgia and hysterectomy: the impact on health status and health care costs. Clin Rheumatol. 2012;31(11):1585-1589.

34. Izquierdo-Alvarez S, Bocos-Terraz JP, Bancalero-Flores JL, Pavon-Romero L, Serrano-Ostariz E, de Miquel CA. Is there an association between fibromyalgia and below-normal levels of urinary cortisol? BMC Res Notes. 2008;1:134.

35. Korszun A, Young EA, Engleberg NC, et al. Follicular phase hypothalamic-pituitary-gonadal axis function in women with fibromyalgia and chronic fatigue syndrome. J Rheumatol. 2000;27(6):1526-1530.

36. McLean SA, Williams DA, Harris RE, et al. Momentary relationship between cortisol secretion and symptoms in patients with fibromyalgia. Arthritis Rheum. 2005;52(11):3660-3669.

37. McBeth J, Chiu YH, Silman AJ, et al. Hypothalamic-pituitary-adrenal stress axis function and the relationship with chronic widespread pain and its antecedents. Arthritis Res Ther. 2005;7(5):R992-R1000.

38. Bazzichi L, Rossi A, Zirafa C, et al. Thyroid autoimmunity may represent a predisposition for the development of fibromyalgia? Rheumatol Int. 2012;32(2):335-341.

39. Garrison RL, Breeding PC. A metabolic basis for fibromyalgia and its related disorders: the possible role of resistance to thyroid hormone. Med Hypotheses. 2003;61(2):182-189.

40. Soy M, Guldiken S, Arikan E, Altun BU, Tugrul A. Frequency of rheumatic diseases in patients with autoimmune thyroid disease. Rheumatol Int. 2007;27(6):575-577.

41. Ribeiro LS, Proietti FA. Interrelations between fibromyalgia, thyroid autoantibodies, and depression. J Rheumatol. 2004;31(10): 2036-2040.

42. Gur A, Cevik R, Nas K, Colpan L, Sarac S. Cortisol and hypothalamicpituitary-gonadal axis hormones in follicular-phase women with fibromyalgia and chronic fatigue syndrome and effect of depressive symptoms on these hormones. Arthritis Res Ther. 2004;6(3):R232-R238.

43. Gur A, Cevik R, Sarac AJ, Colpan L, Em S. Hypothalamicpituitary-gonadal axis and cortisol in young women with primary fibromyalgia: the potential roles of depression, fatigue, and sleep disturbance in the occurrence of hypocortisolism. Ann Rheum Dis. 2004;63(11):1504-1506.

44. Wingenfeld K, Wagner D, Schmidt I, Meinlschmidt G, Hellhammer DH, Heim C. The low-dose dexamethasone suppression test in fibromyalgia. J Psychosom Res. 2007;62(1):85-91.

45. Wingenfeld K, Heim C, Schmidt I, Wagner D, Meinlschmidt G, Hellhammer DH. HPA axis reactivity and lymphocyte glucocorticoid sensitivity in fibromyalgia syndrome and chronic pelvic pain. Psychosom Med. 2008;70(1):65-72.

46. Bonifazi M, Suman AL, Cambiaggi C, et al. Changes in salivary cortisol and corticosteroid receptor-alpha mRNA expression following a 3-week multidisciplinary treatment program in patients with fibromyalgia. Psychoneuroendocrinology. 2006;31(9):1076-1086. 
47. Wolfe F, Smythe HA, Yunus MB, et al. The American College of Rheumatology 1990 Criteria for the Classification of Fibromyalgia. Report of the Multicenter Criteria Committee. Arthritis Rheum. 1990; 33(2):160-172.

48. Giacomelli C, Talarico R, Bombardieri S, Bazzichi L. The interaction between autoimmune diseases and fibromyalgia: risk, disease course and management. Expert Rev Clin Immunol. 2013;9(11):1069-1076.

49. Whiteman MK, Hillis SD, Jamieson DJ, et al. Inpatient hysterectomy surveillance in the United States, 2000-2004. Am J Obstet Gynecol. 2008;198(1):34. e31-e37.

50. Keshavarz H, Hillis SD, Kieke BA, Marchbanks PA. Hysterectomy surveillance - United States, 1994-1999. MMWR Morb Mortal Wkly Rep. 2002;51(S05):1-8.

51. Albano C, Platteau P, Devroey P. Gonadotropin-releasing hormone antagonist: how good is the new hope? Curr Opin Obstet Gynecol. 2001;13(3):257-262

52. Glintborg D, Andersen M. An update on the pathogenesis, inflammation, and metabolism in hirsutism and polycystic ovary syndrome. Gynecol Endocrinol. 2010;26(4):281-296.

53. Gillespie CF, Nemeroff CB. Hypercortisolemia and depression. Psychosom Med. 2005;(67 Suppl 1):S26-S28.

54. Arborelius L, Owens MJ, Plotsky PM, Nemeroff CB. The role of corticotropin-releasing factor in depression and anxiety disorders. J Endocrinol. 1999;160(1):1-12.

55. Tzioufas AG, Tsonis J, Moutsopoulos HM. Neuroendocrine dysfunction in Sjogren's syndrome. Neuroimmunomodulation. 2008;15(1):37-45.

56. Harbuz MS, Richards LJ, Chover-Gonzalez AJ, Marti-Sistac O, Jessop DS. Stress in autoimmune disease models. Ann N Y Acad Sci. 2006;1069:51-61.
57. Silverman MN, Heim CM, Nater UM, Marques AH, Sternberg EM. Neuroendocrine and immune contributors to fatigue. PM R. 2010; 2(5):338-346.

58. Born J, Wagner U. Sleep, hormones, and memory. Obstet Gynecol Clin North Am. 2009;36(4):809-829.

59. Conrad CD, Bimonte-Nelson HA. Impact of the hypothalamicpituitary-adrenal/gonadal axes on trajectory of age-related cognitive decline. Prog Brain Res. 2010;182:31-76.

60. Straub RH. The complex role of estrogens in inflammation. Endocr Rev. 2007;28(5):521-574.

61. Goswami C, Kuhn J, Dina OA, et al. Estrogen destabilizes microtubules through an ion-conductivity-independent TRPV1 pathway. JNeurochem. 2011;117(6):995-1008.

62. Wu YW, Bi YP, Kou XX, et al. 17-Beta-estradiol enhanced allodynia of inflammatory temporomandibular joint through upregulation of hippocampal TRPV1 in ovariectomized rats. J Neurosci. 2010 30(26):8710-8719.

63. Xu S, Cheng Y, Keast JR, Osborne PB. 17beta-estradiol activates estrogen receptor beta-signalling and inhibits transient receptor potential vanilloid receptor 1 activation by capsaicin in adult rat nociceptor neurons. Endocrinology. 2008;149(11):5540-5548.

64. White KP, Speechley M, Harth M, Ostbye T. The London Fibromyalgia Epidemiology Study: the prevalence of fibromyalgia syndrome in London, Ontario. J Rheumatol. 1999;26(7):1570-1576.

65. Wolfe F, Clauw DJ, Fitzcharles MA, et al. The American College of Rheumatology preliminary diagnostic criteria for fibromyalgia and measurement of symptom severity. Arthritis Care Res (Hoboken). 2010; 62(5):600-610.
Journal of Pain Research

\section{Publish your work in this journal}

The Journal of Pain Research is an international, peer-reviewed, open access, online journal that welcomes laboratory and clinical findings in the fields of pain research and the prevention and management of pain. Original research, reviews, symposium reports, hypothesis formation and commentaries are all considered for publication.

\section{Dovepress}

The manuscript management system is completely online and includes a very quick and fair peer-review system, which is all easy to use. Visit http://www.dovepress.com/testimonials.php to read real quotes from published authors. 